\title{
COVID-19 anxiety scale (CAS): Development and psychometric properties
}

\author{
Washington Allysson Dantas Silva ${ }^{1,2}$ (D) $\cdot$ Tátila Rayane de Sampaio Brito ${ }^{1}$ (D) $\cdot$ Cicero Roberto Pereira ${ }^{1}$ (D)
}

Accepted: 11 November 2020 / Published online: 13 November 2020

(C) Springer Science+Business Media, LLC, part of Springer Nature 2020

\begin{abstract}
The objective of the present research is to develop and validate the COVID-19 Anxiety Scale (CAS). We conducted three studies to gather evidence regarding content and construct validity, as well to evaluate the reliability of the measure. Study 1 is subdivided into two studies. In Study 1a, we analyze the content validity of the new measure through content expert analysis $(N=10$ expert raters). In Study $1 \mathrm{~b}(N=30)$, we perform a pilot study with a sample from the target population. In Study $2(N=$ 352 ), we explored the unifactorial structure of the measure, and analyzed its internal consistency verified. Finally, in Study $3(N=$ 200), we assess the adequacy of the factor structure and gather evidence on convergent-discriminant validity and gender-scale invariance. In summary, our results demonstrate that CAS is a reliable and adequate instrument to assess COVID-19 related anxiety.
\end{abstract}

Keywords COVID-19 $\cdot$ Anxiety $\cdot$ Pandemic $\cdot$ Measures

\section{Introduction}

The impacts of the new Coronavirus pandemic (SARS-Cov2) on the population's mental health is one of the greatest challenges faced by humanity in the twenty-first century. The virus was first identified in the city of Wuhan, China, and has been recognized as the cause of Severe Acute Respiratory Syndrome, under the acronym COVID-19 (Corona Virus Disease 2019). Due to the exponential increase in the number of cases, the speed of infection, and the number of deaths by the disease, the World Health Organization declared a state of pandemic in March 2020 (WHO. World Health Organization, 2020). To exemplify the rapid proliferation of the virus and the infection's effects on the body, on October 27st in Brazil, 15.726 new cases were reported throughout the national territory, totaling almost six millions confirmed cases and 150 thousand deaths, prompting the disease to be considered the main cause of deaths in the country (Brazil. Health Ministry, 2020). Therefore, there is a critical

Washington Allysson Dantas Silva

allysson_dantas@hotmail.com

1 Present address: Departament of Psychology, Universidade Federal da Paraíba, João Pessoa, PB, Brazil

2 João Pessoa, Brazil social context creating the optimal conditions for the emergence of psychological factors, affecting people's quality of life and psychological well-being.

Studies have shown that crisis situations, such as the new coronavirus pandemic, have a negative impact on individual's mental health (Kang et al., 2020; Mukhtar, 2020). The extreme concern about the possibility of becoming infected with COVID-19, or the imminence of the idea of death itself, can be intensified during the pandemic period (Pakpour \& Griffiths, 2020). Such excessive concern may lead people to change their psychological functioning, by emphasizing the negative aspects of the issue and the frequent thoughts on the subject, which could affect their psychological well-being (Wang et al., 2020a; Zandifar \& Badrfam, 2020). Along this line of thinking, we ask ourselves: does living through the pandemic period impact individuals' anxiety? To answer this question, a measure able to accurately assess the fluctuations in anxiety levels caused by COVID-19 is essential. To elaborate this instrument, we rely on the operational and constitutive definition of the Generalized Anxiety Disorder symptoms, which - according to the Diagnostic and Statistical Manual of Mental Disorders - DSM-V (APA. American Psychiatric Association, 2013) - includes a type of concern that is difficult to control, persistent and excessive, along with the following symptoms: restlessness or feeling on edge, tiredness, difficulty concentrating, irritability, muscle tension, sleep disturbance, and a decreased ability to engage in social 
activities. These symptoms do not derive from organic pathologies or the use of psychoactive substances.

In the present research, we are led by this definition, adapting it to contemplate a specific event that has generated excessive concern globally. Hence, we focus on a specific type of anxiety caused by the pandemic scenario: COVID-19 anxiety. Although studies have already been conducted regarding the consequences for the general psychological well-being due to social distancing, resulting from the COVID-19 prevention (Wang et al., 2020b), to date few instruments have been built - or adapted - to assess the psychological demands regarding COVID-19 (Ahorsu et al., 2020; Oliver, Barber, Roomp, \& Roomp, 2020; Taylor et al., 2020). More objectively, among those instruments already built, there is no one validated for the Brazilian context, that is able to evaluate the contextual COVID-19 anxiety. Thus, we seek to contribute by filling this gap and offering an instrument able to gauge fluctuations in the levels of COVID-19 anxiety, given the relevance and urgency of the matter. Therefore, we have developed a set of items through which we may adequately assess this type of anxiety.

\section{Overview of Studies}

Through the course of three studies, we have developed and presented evidence on the validity and reliability of the COVID-19 Anxiety Scale (CAS), in addition to demonstrating that such scale is equally suitable for assessing the anxiety of men and women in the Brazilian context. In Study 1, we developed a pool of items and evaluated the CAS content validity by means of a content expert validation (Study 1a), and the evaluation of the items' comprehensibility (Study 1b). In Study 2, based on a sample of the Brazilian population, we explored whether the CAS presents a single-factor structure. Finally, in Study 3, we applied a set of Confirmatory Factor Analyzes to test the hypothesis regarding the onedimensionality of the CAS items, as well as to analyze their configural, metric, and scalar invariance by gender. Furthermore, we explore its convergent and discriminant validity with other instruments which, theoretically, measure similar and different constructs of anxiety. In all studies, participants were informed about all relevant aspects of the study before they started to fill out the questionnaire. We guaranteed their anonymity, privacy, and confidentiality, according to the ethical standards and procedures for research with human beings (World Medical Association, 2001; Brazil. Health Ministry, 2012, 2016). The inclusion criteria for participants in all studies were: being a Brazilian citizen, residing in any region of the country, and being of legal age (older than 18). The research protocol was approved by the local Ethics Committee of the first author's institution.

\section{Study 1: CAS Content Development and Validity}

In this first study, we detail the process of preparing the CAS items, examining their content validity by means of a content expert validation (Study 1a), and assessing the comprehensibility of the items by the target population (Study 1b). For the development of the scale items, we followed the guidelines for the construction of psychological instruments (Nunnally \& Bernstein, 1994). First, we apply the definition of DSM-V for the construct operationalization as a basis. We carried out an exhaustive reading and then listed the main points to ensure that the set of items was sparing. We then tailor the items to the specific object, i.e. the COVID-19 pandemic. After this stage, we defined the target group, which was composed of adults from the general Brazilian population. Subsequently, we decided on the type of instrument to be applied, which was defined as a self-reporting measurement tool. Finally, after these steps, we proceeded to the development of the CAS items. Aiming for the consistency of the items in order to assess the defined construct, we elaborated a set of statements, which had previously been the object of analysis for their conceptual adequacy by the first two authors.

After the elaboration, the third author reanalyzed the content of each item to confirm its adequacy in relation to the concept of anxiety employed here. From this analysis, statements with identical contents were removed, resulting in an initial version consisting of 7 items. Finally, we proceeded to the content validity stage submitting the set of items for evaluation by expert judges in the field of anxiety studies.

\section{Study 1a: Content Validity}

\section{Participants}

For the content expert validation, we consulted 10 Psychology professionals with experience in the construction and adaptation of psychological instruments. Nine were women and one was a man, with ages ranging from 23 to 43 years $(M=27.75, S D=$ $6.77)$ and average education history of 4.12 years $(S D=4.7)$.

\section{Measures}

The panel of experts received an online survey containing the operational definition of the construct (e.g., anxiety), the items proposed for the scale (Table 1). The content experts are then asked to examine the items according to three criteria (Grant \& Davis, 1997): representativeness (the degree which the item reflects the operationalized construct), relevance (importance of the item to explain the construct) and clarity (accessibility for comprehensiveness). This panel's duty was to sort the items telling us "how much" each item measures each category in the 6-point rating scale ranging from 0 (not 
Table 1 7-item of CAS

Instructions: How much each item reflected their behavior in the last days? Please, indicate using the scale $(0=$ not applicable to me, $1=$ hardly ever applicable to me, $2=$ sometimes applicable to me, and $3=$ very applicable to me).

\begin{tabular}{ll} 
No. & Items \\
\hline 1. & I feel bad when thinking about COVID-19 \\
2. & I feel heart racing when I read about COVID-19 \\
3. & I feel anxious about COVID-19 \\
4. & I feel uneasy when reading news about COVID-19 \\
5. & I have trouble relaxing when I think about COVID-19 \\
6. & I feel like I may panic when I update myself about COVID-19 \\
7. & I am afraid of being infected with COVID-19 \\
\hline
\end{tabular}

representative or not relevant or not clear) to 5 (very representative or very relevant or very clear). Thus, the higher the grade, the more pertinent and/or relevant and/or clear the item was considered to assess the construct.

\section{Procedures}

We invited the content experts via email, informing the objectives of the scale and the number of proposed items. Post acceptance, we forwarded both the Informed Consent Form (ICF), informing them of the ethical guidelines for research with human beings, and the online questionnaire for the scale evaluation.

\section{Data Analysis}

To verify the content expert validation, we calculated the judgment-quantification by Content Validity Coefficient (CVC) (Aiken, 1980). More specifically, we calculated the $\mathrm{CVC}$ for content expert's judgment $(\mathrm{CVCj})$, for each item (CVCi), and for the total scale (CVCt), using CVC values $\geq .80$ as the criterion for content validity (Aiken, 1985).

\section{Results}

The results demonstrated that all items presented values above .80 regarding pertinence, relevance, and clarity. Additionally, the content expert's judgment for each item $(\mathrm{CVCj})$ ranged from .94 to 1.00. Finally, the CVC value for the scale (CVCt) was 97 .

\section{Study 2a: Examining the Item's Comprehensibility}

We conducted a pilot study with a small sample of Brazilians over 18 years of age to verify the comprehensibility of each item by the target population. This is an important step in the validation of an instrument, as it aims to ensure that the written items are clearly and comprehensively by the participants.

\section{Participants}

Thirty individuals from the general population participated in this pilot study, 15 men and 15 women, aged between 18 and 65 years old $(M=27.83, S D=9.01)$, in its majority single $(86.7 \%)$ and self-declared heterosexuals $(53.3 \%)$.

\section{Measures}

We administered the 7-item initial version of COVID-19 Anxiety Scale (CAS).

\section{Procedures}

After being assigned the ICF, participants were asked to evaluate the comprehension of each item using a 4-point rating scale $(0=$ lack of comprehension and $3=$ total comprehension). If a participant indicated difficulties in understanding the item (by checking an option below 3 on the scale), they were asked to indicate on how the item could be improved to become more comprehensive.

\section{Data Analysis}

We analyzed the data using the IBM SPPS (Statistical Package for the Social Sciences, version 24) software. We considered the maximum agreement among the participants as criterion for the items' comprehensibility. That is, the average of the participants should be equal to 3.0 , with a standard deviation of 0.0. Otherwise, the item would be considered partially inappropriate (Wong \& Chow, 2017).

\section{Results}

The results demonstrated that all participants marked the maximum scale option for each of the 7 proposed items $(M=3.00$, $S D=.00)$. Namely, they demonstrated a high comprehensibility of all items, indicating that there would be no need for reformulation.

\section{Discussion}

In this study, we developed the CAS items and analyzed their content validity through judgment-quantification (Study 1a), and by reviewing the comprehensibility of the scale items (Study 1b). The results revealed that the seven items we elaborated from the definition of anxiety indicated by the DSM-V were judged as adequate by the content experts and as comprehensive by exemplars of the target population. In 
summary, these results evidence the content validity of these items to measure anxiety associated with COVID-19. However, we need to verify the quality of the factor structure by addressing the quality of the items using a more extensive and diverse sample.

\section{Study 2: Exploratory Factor Analysis of CAS}

The goal of this study was to gather initial evidence of the CAS factor validity and its reliability. In this analysis, we seek to explore the factor structure of the COVID-19 Anxiety Scale administrating the items on a sample of the general Brazilian population.

\section{Method}

\section{Participants}

Three hundred and fifty-two individuals participated, mostly female $(71 \%)$, aged between 18 and 47 years of age $(M=$ $29.56, S D=9.97)$, mainly single $(68.5 \%)$ and predominantly heterosexual $(74.7 \%)$.

\section{Measures}

We administered the COVID-19 Anxiety Scale (CAS) developed in Study 1. The participants indicated how much each item reflected their behavior in the last days, always regarding COVID-19, using a 4-point scale ( $0=$ not applicable to me and $3=$ very applicable to me). The level of anxiety was measured by averaging the participants' scores (ranging from 0 to 3 ), so that the higher the average, the greater the anxiety of the individual regarding COVID-19.

\section{Procedures}

We collected data online using Qualtrics platform. Participants were invited through posts on social media, where a link to the questionnaire was made available. The first page of this questionnaire contained the Informed Consent Form (ICF), comprising information regarding the research objectives and how to contact the responsible researcher. Participants had access to the questionnaire only if they consented and agreed to the ICF. The questionnaire took approximately five minutes to fill in.

\section{Data Analysis}

We analyzed the data using the IBM SPSS and JASP (version 0.11.1.0) software. We estimated descriptive statistics, bivariate correlation coefficients and parametric tests for differences in means. For scale's factorial structure, we calculated an Exploratory Factor Analysis using the Principal Axis Factoring extraction method. We adopted the Kaiser-Guttman criterion (Kaiser, 1960) for the retention of factors, i.e., eigenvalues equal to or greater than 1.00 . We consider as adequate loading factor equal to or greater than .35 to retain the item on the scale (Hair, Black, Babin, Anderson, \& Tatham, 2006). For internal consistency, we calculated the Cronbach's alpha $(\alpha)$ and McDonald's omega $(\omega)$ coefficients, assuming values equal to or greater than .70 as acceptable.

\section{Results}

Initially, we analyzed the adequacy of the sample and the correlation matrix for the use of an Exploratory Factor Analysis (EFA). The results of the Kaiser-Meyer-Olkin Measure $(K M O=.89)$ and Bartlett's Sphericity $\left[\chi^{2}(21)=\right.$ $1382.14, p<.001]$ tests demonstrated the sample adequacy, and the scale could be factorable. The results (Table 2) demonstrated the extraction of a single factor, with an eigenvalue of 4.34 , which explained $62.02 \%$ of the variance. The factorial loadings ranged from .53 (e.g., I am afraid of becoming infected with COVID-19) to .88 (e.g., I have trouble relaxing when I think about COVID-19). Cronbach's alpha and McDonald's omega values were .89 each.

Subsequently, we analyzed the relation between anxiety measured with the CAS and the sociodemographic characteristics of the participants. We found no significant relationship between age and anxiety $(r=-.02, p=.63)$, no significant differences by marital status $[F(5346)=.455, p=.81]$ or by sexual orientation $[F(3348)=1.98, p=.11]$. Only the averages of anxiety between men and women proved to be statistically different $[t(347)=-3.95, p<.001, d=-.47]$. Overall, female participants $(M=2.59, S D=.81)$ presented higher levels of anxiety regarding COVID-19 than men $(M=2.21$, $S D=.77)$.

\section{Discussion}

In this study we obtained preliminary evidence of CAS factor validity and reliability. The main results revealed that the scale items measure a single factor structure, with adequate psychometric indicators (e.g., factorial loads). In addition, the observed reliability coefficients indicate that its internal consistency is strong. As demonstrated by previous research on anxiety in general (Sharma, Powers, Bradley, \& Ressler, 2016), women's anxiety levels regarding COVID-19 were higher than those presented by men. Therefore, the CAS is sensitive enough to detect differences in the levels of anxiety experienced by men and women. However, the quality and relevance of this type of comparison is dependent on whether CAS evaluates anxiety in an equivalent manner between both genders. 
Table 2 Exploratory Factor Analysis of CAS

\begin{tabular}{lllll}
\hline No. & Items & $\mathrm{M}(\mathrm{SD})$ & Factor loadings & $\mathrm{h}^{2}$ \\
\hline 05. & I have trouble relaxing when I think about COVID-19 & $1.37(1.13)$ & .88 & .78 \\
03. & I feel anxious about COVID-19 & $1.68(1.07)$ & .80 & .64 \\
04. & I feel uneasy when reading news about COVID-19 & $1.77(1.03)$ & .79 & .63 \\
06. & I feel like I may panic when I update myself about COVID-19 & $.92(1.09)$ & .76 & .58 \\
01. & I feel bad when thinking about COVID-19 & $1.89(.95)$ & .72 & .52 \\
02. & I feel heart racing when I read about COVID-19 & $.73(.94)$ & .68 & .47 \\
07. & I am afraid of being infected with COVID-19 & $2.03(1.02)$ & .53 & .28 \\
& Eigenvalue & & 4.32 & \\
& \% Variance & & 62.02 & \\
\hline
\end{tabular}

Note. $\mathrm{h}^{2}=$ communality
Therefore, it will be necessary not only to confirm the onedimensionality of the measure, but also its configural, metric, and scalar invariance by gender of participants.

\section{Study 3: CAS Factor and Convergent-Discriminant Validity}

We carried out a new study to confirm the unifactorial structure of CAS. Moreover, we explore its convergent and discriminant validity by proposing the hypothesis that the CAS is positively related to other measures that evaluate anxiety, but it is not related to different construct scales. In addition, to ensure the structure, factorial loadings and intercepts presented by the scale are valid for both men and women, we analyzed the configural, metric, and scalar invariance of CAS according to the gender of participants.

\section{Method}

\section{Participants}

Two hundred individuals from the general Brazilian population participated in this study, 102 women and 98 men, aged between 18 and 53 years old $(M=25.0, S D=6.69)$.

\section{Measures}

As in Study 2, participants completed an online questionnaire consisting of sociodemographic items (e.g., age, sex, marital status, and sexual orientation) and the following scales:

COVID-19 Anxiety Scale (CAS) We applied the same version of the previous study. Participants responded to the seven items indicating how much each applied to their behavior in the past few days regarding COVID-19 using a 4-point scale $(0=$ not applicable to me and $3=$ very applicable to $\mathrm{me}$ ).
Depression Anxiety and Stress Scale (DASS-21) To assess the CAS convergent validity, we applied the reduced version of DASS-21 (Lovibond \& Lovibond, 1995). The reduced version of the scale was adapted for Brazilian Portuguese by Vignola and Tucci (2014), being an instrument composed of 21 items in a 4-point scale $(0=$ not applicable to me and $3=$ very applicable to me). In the current study, participants answered only the Anxiety dimension ( 7 items), as we sought to verify whether CAS would be related to a measure built to evaluate the same construct. Examples of items in this dimension are: "I felt trembly (for example, my hands)" and "I felt I was going to panic." In this study, this dimension had a high internal consistency $(\alpha=.86, \omega=.86)$.

Intergroup Differentiation Scale To examine the CAS discriminant validity, we used the Brazilian Portuguese translation of the Intergroup Differentiation Scale (FalomirPichastor, Mugny, \& Berent, 2017). This measurement assesses personal motivation to differentiate a group from a relevant outgroup, and it is expected that this motivation is not related to the anxiety regarding COVID-19. The five items on the scale are based on essentialist beliefs regarding psychological differences between heterosexual and gay people in general, exemplified in "gay people and heterosexuals are psychologically different " and "gay people and heterosexuals have different emotional characteristics ". The item response is a 4-point Likert scale ( $1=$ strongly disagree to $7=$ strongly agree). In order to verify the quality of the factor structure of the measure in the present study, we performed an EFA by principal axis factoring method, which results demonstrated the existence of a single-factor (eigenvalue $=3.14$, explained variance $=62.94 \%$, factor loadings .80 to .57$)$. Also, the measure showed high internal consistency $(\alpha=.85, \omega=.85)$.

\section{Procedures}

For data collection, we proceeded in the same manner as in Study 2, by organizing the instruments in an online 
questionnaire and inviting people to participate in the survey through publications on social media (e.g., WhatsApp, Instagram and Facebook). The questionnaire took approximately ten minutes to fill in.

\section{Data Analysis}

We performed the Confirmatory Factor Analysis (CFA) using Mplus (v. 8.3, Muthén \& Muthén, 2017) using the maximum likelihood (ML) as estimator, applying the following indicators as quality of fit parameters: the chi-square test by the degree of freedom $\left(\chi^{2} / \mathrm{gl}\right)$ lower than 5; comparative fit index (CFI) and Tucker-Lewis index (TLI), both greater than .95 (Bentler, 1990; Tucker \& Lewis, 1973); root-mean-squareerror of approximation (RMSEA) and standardized rootmean-square residual (SRMR) lower than .050 as an indication of optimal fit, and lower than .08 as an acceptable fit (Hu $\&$ Bentler, 1999). For the analysis of the CAS convergent and discriminant validity, we used the IBM SPSS software (Statistical Package for the Social Sciences, version 24). In this software, we correlated the anxiety measure by CAS with the DASS-21 Anxiety dimension (convergent measure) and the Intergroup Differentiation Scale (discriminant measure). For convergent and discriminant validity, we expected that CAS should correlate, in a moderate and significant way $(r>.50)$ with the convergent measure, and not correlate (or weakly correlate) with the convergent measure $(r<.20)$. Furthermore, we calculated the gender invariance using JASP (version 0.11.1.0), applying the Multi-group Confirmatory Factor Analysis (MGCFA), established under three analysis models: the configural, metric, and scalar invariance. The configural invariance tests whether the factorial structure of the scale is equivalent in both groups. The metric invariance analyzes whether the factorial loads are equivalent for men and women. Finally, the scalar invariance assesses whether the intercepts, or mean scores of the latent variable, are the same for both groups (Putnik \& Bornstein, 2016). Therefore, based on the guidelines suggested by Chen (2007), we used Delta CFI $(\Delta \mathrm{CFI} \leq .01)$ and Delta RMSEA $(\triangle \mathrm{RMSEA} \leq .015)$ as invariance rejection parameters.

\section{Results}

Table 3 presents the descriptive statistics of the items and the total CAS score. As in the previous study, we found no significant relationship between age $(r=-.08, p=.24)$, marital status $[F(4.195)=.21, p=.93]$, and sexual orientation $[F$ $(3.196)=1.21, p=.30]$ and CAS scores. Only the gender difference between was significant $[t(198)=-3.77, p<.001$, $d=-.53]$. Women $(M=2.10, S D=.59)$ had higher scores of anxiety associated with COVID-19 than men $(M=1.75$, $S D=.70)$, with this difference being significant. The internal consistency of CAS was high $(\alpha=.86, \omega=.86)$.
We estimated the CFA by specifying a single-factor model, which resulted in the following indices: $\chi^{2} / \mathrm{gl}=1.89$, $\mathrm{CFI}=.97, \mathrm{TLI}=.96, \mathrm{RMSEA}=.06(90 \% C I=.02 ; .10)$ and $\mathrm{SRMR}=.03$. Except for RMSEA, these results demonstrated that the unifactorial model fits very well to the data. The factorial loadings of the items ranged from .40 (e.g., I am afraid of becoming infected with COVID-19) to .85 (e.g., I have trouble relaxing when I think about COVID-19), all of which are statistically significant. $(p<.001)$, as illustrated in Fig. 1.

We then explored the CAS convergent and discriminant validity. The results showed a high and significant correlation between CAS and the DASS-21 Anxiety dimension $(r=.65$, $p<.01)$, and a non-significant correlation with the Intergroup Differentiation Scale $(r=.09, p=.19)$.

Finally, we examined the gender invariance, considering the groups of men and women (Table 4). The MGCFA results demonstrated the configural, metric, and scalar invariance between the gender groups $(\Delta \mathrm{CFI} \leq .01$ and $\triangle \mathrm{RMSEA} \leq .015)$.

\section{Discussion}

The results of this study showed additional evidence of the validity of the CAS single-factor structure. The internal consistency estimates also confirmed the good reliability of the seven-item version. As predicted, the scale correlated with the anxiety measure, and it did not correlate with the Intergroup Differentiation Scale, which is consistent with the CAS convergent and discriminant validity hypotheses. Moreover, results showed the configural, metric and scalar invariance of CAS between participants' gender. As in the previous study, women present higher levels of anxiety than men, a circumstance that is important to be evaluated when conducting future studies.

Table 3 Descriptive statistics of CAS items

\begin{tabular}{lllll}
\hline Items & \multicolumn{3}{l}{$\mathrm{M}(\mathrm{SD})$} & \\
\cline { 2 - 4 } & Total & Men & Women & $\mathrm{p} *$ \\
\hline 1 & $2.34(.79)$ & $2.22(.79)$ & $2.45(.79)$ & .043 \\
2 & $1.01(.94)$ & $.76(.87)$ & $1.25(.95)$ & .001 \\
3 & $2.26(.85)$ & $2.06(.95)$ & $2.46(.71)$ & .001 \\
4 & $2.16(.88)$ & $2.00(.94)$ & $2.37(.78)$ & .003 \\
5 & $2.06(.98)$ & $1.83(1.05)$ & $2.27(.86)$ & .002 \\
6 & $1.29(1.05)$ & $1.10(1.02)$ & $1.47(1.06)$ & .014 \\
7 & $2.39(.82)$ & $2.31(.84)$ & $2.46(.80)$ & .217 \\
Total score & $1.93(.66)$ & $1.75(.70)$ & $2.10(.59)$ & .001 \\
\hline
\end{tabular}

Note. ${ }^{*}=$ independent-sample t test $(p$ value $)$ 
Fig. 1 Confirmatory Factor Analysis of CAS items

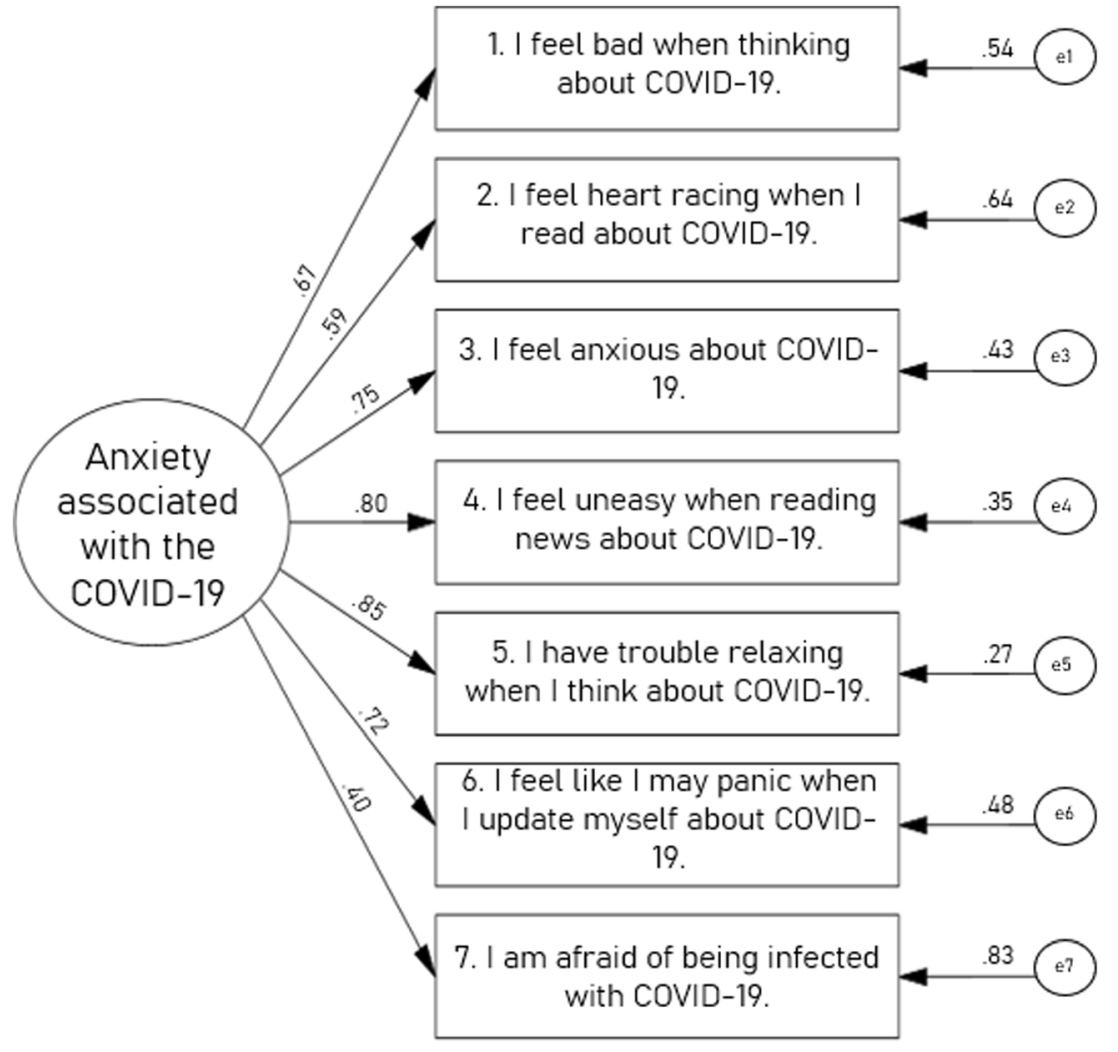

\section{General Discussion}

This research program sought to develop and gather evidence on the validity and reliability of the COVID-19 Anxiety Scale (CAS). Study 1 evaluated the content validity of the proposed measure; Study 2 explored its single-factor structure; and Study 3 confirmed its unifactorial structure, as well gathering evidence of convergent and discriminant validity. Applied together, the results of the three studies support the use of CAS as a measure that is appropriate and very simple to apply in order to assess anxiety associated with the COVID-19 pandemic.

With regard to content validity (Grant \& Davis, 1997), the present research demonstrated that CAS is adequate to measure what it intends to evaluate, that is, its items are consistent with the operational definition of anxiety associated with COVID-19. We also gathered evidence on the factor validity (Nunnally \& Bernstein, 1994) of the CAS. More specifically, we proved that it is a unidimensional measure with high internal consistency. Furthermore, the positive, strong, and significant relationship between CAS and another measure of anxiety offers additional support to its construct validity. Moreover, the instrument was not related to a measure that assesses a psychological construct other than anxiety, guaranteeing the discriminatory validity of the CAS. Additional evidence of validity was also identified, specifically the configural, metric, and scalar invariance between different gender groups.

Furthermore, we examined whether the CAS participants' scores differed across socio-demographic variables, revealing that only gender differences in individual's anxiety during the pandemic. These results are consistent with previous research (Alon, Doepke, Olmstead-Rumsey, \& Tertilt, 2020; Wenham, Smith, \& Morgan, 2020) that have already shown the impact of gender in crisis situations. Women are exposed to several psychological vulnerability factors that may aggravate the levels of anxiety associated with COVID-19, such as domestic violence (Garcia-Moreno, Heise, Jansen, Ellsberg, \& Watts, 2005), work overload (Mittal \& Bhakar, 2018), and discrimination (Parashar, 2020). The CAS proved to be sufficiently sensitive to assess these differences, which had been observed in those studies.

The understanding of anxiety as a unidimensional construct has been prevalent throughout the psychological literature (Francis, Noel, \& Ryan, 2019; Olatunji \& WolitzkyTaylor, 2009). Our results are consistent with this approach and conceive anxiety as a persistent and excessive concern that is usually accompanied by physical symptoms, such as muscle tension, heart racing, sweating, and insomnia. More specifically, we address a particular type of anxiety, which is that associated with COVID-19. Therefore, we offer a psychometrically suitable measurement tool to assess this type of psychological state. 
Table 4 Goodness-of-fit indices for invariance analysis of the CAS single-factor model between gender groups

\begin{tabular}{llllllll}
\hline Model & $\chi^{2}$ & $d f$ & $\begin{array}{l}\chi^{2} / \\
d f\end{array}$ & CFI & $\Delta$ CFI & RMSEA(90\%CI) & $\Delta$ RMSEA \\
\hline Configural & 37.84 & 28 & 1.35 & .982 & - & $.059(.000-.104)$ & - \\
Men & 20.74 & 14 & 1.48 & .979 & - & $.070(.000-.130)$ & - \\
Women & 17.10 & 14 & 1.22 & .986 & - & $.047(.000-.112)$ & \\
Metric & 47.84 & 34 & 1.40 & .974 & .008 & $.064(.000-.103)$ & .005 \\
Scalar & 52.62 & 40 & 1.31 & .977 & .003 & $.056(.000-.094)$ & .008 \\
\hline
\end{tabular}

Note $. \mathrm{df}=$ degrees of freedom; CFI $=$ Comparative Fit Index; RMSEA = Root Mean Square Error of Approximation; $\mathrm{CI}=$ Confidence Interval
It is important to publish an article on a scale that assesses anxiety associated with COVID-19 for several reasons. First, there is evidence that the current pandemic caused pervasive consequences for people's mental health. With alarming rates of increase in cases of anxiety symptoms, potentiation of fears about the future, and uncertainties common to periods of adversity that question people's stability and sense of security about their lives, there is a need for valid and accurate tools to assess the fluctuations in anxiety caused by the specific situation of the pandemic. Therefore, it is extremely relevant to develop valid and reliable psychological instruments to measure as individuals are reacting to this situation. Second, results from CAS may help to subsidize the development of public policies aimed to deal directly with the promotion of psychological well-being, such as psychoeducation actions that have a preventive character, as well as, to guide interventions in the treatment of anxiety associated to COVID19. Third, the results of research on anxiety during the pandemic can be useful as a parameter for the understanding of similar events that may occur in the future. For instance, the scale has the potential to be used after the current pandemic since it can easily be adapted by replacing the term "COVID-19" by other terms referring to adverse situations in further studies. Finally, the CAS differs from other scales of anxiety, firstly, by placing the anxious symptomatology in a specific context by focusing on the COVID-19 pandemic as a particular source of anxiety. Second, the CAS differs from other scales by being based on the operational definition proposed in the DSM-V. Specifically, it takes as a basis for the elaboration of the relevant behavioral items and episodes that generate anxious symptoms, such as extreme adverse situations, such as the COVID-19 pandemic.

Although this research presents important contributions, it is not without limitations. First, it was not possible to use clinical samples, which demonstrates the criterion validity since this sample has higher scores than non-clinical samples. We suggest future studies to be carried out to fill this gap. Furthermore, we did not assess whether the CAS is sensitive to contextual fluctuations that cause a momentary increase or decrease in anxiety, which could be demonstrated in experimental studies manipulating causal factors of this anxiety, such as the prominence of fear of death (e.g., Klackl \& Jonas, 2019). The scores obtained here are ex-post-facto, that is, the levels of anxiety had already been impacted by other factors prior to the moment of data collection. Also, some items included the word feel in this formulation. In this regard, expressions using the word "feel" can be especially appreciated by people with a high level of need to affect the consequences of the so-called correspondence effect (Aquino et al., 2020; Clarkson, Tormala, \& Rucker, 2011). We tested the correlations among the errors of items, and we observed that some of them were correlated. However, this possibility did not decrease the quality of the model fit, nor did it decrease the reliability of the estimated parameters. Finally, we did not assess heterogeneous samples, of different age groups, as well as those constituting risk groups (e.g., hypertensive, diabetic, people with cancer, and the elderly).

Despite these limitations, the results we found are strong enough to show evidence of the CAS content, factor, and construct validity, ensuring its use in research aimed at assessing the COVID-19 related anxiety levels. This instrument may contribute to further research on individual's psychological reactions to pandemic situations, and it may also assist in decision making in other social contexts, since the concern with the impacts of the pandemic extends to the most diverse social spheres.

Data Availability Statements The datasets generated during and/or analysed during the current study are available in the OPEN SCIENCE FRAMEWORK repository, https://OSF.IO/JV6KC/.

\section{Compliance with Ethical Standards}

Conflict of Interest We declare that this research has no conflicts of interest.

Ethical Statement and Informed Consent All participants provided consent according to the Declaration of Helsinki and the American Psychological Association. The Research Ethics Committee of Federal University of Paraiba, Brazil approved all procedures used in the studies (CAAE 89390918.0.0000.5188). 


\section{References}

Ahorsu, D. K., Lin, C. Y., Imani, V., Saffari, M., Griffiths, M. D., \& Pakpour, A. H. (2020). The fear of COVID-19 scale: Development and initial validation. International Journal of Mental Health and Addiction. https://doi.org/10.1007/s11469-02000270-8.

Aiken, L. R. (1980). Content validity and reliability of single items or questionnaires. Educational and Psychological Measurement, 40(4), 955-959. https://doi.org/10.1177/001316448004000419.

Aiken, L. R. (1985). Three coefficients for analyzing the reliability, and validity of ratings. Educational and Psychological Measurement, 45(1), 131-142. https://doi.org/10.1177/0013164485451012.

Alon, T. M., Doepke, M., Olmstead-Rumsey, J., \& Tertilt, M. (2020). The impact of COVID-19 on gender equality. National Bureau of Economic Research, 10(24), 16-22. https://doi.org/10.3386/ w26947.

APA. American Psychiatric Association. (2013). Diagnostic and statistical manual of mental disorders - DSM (5th edition). Washington: APA.

Aquino, A., Alparone, F. R., Pagliaro, S., Haddock, G., Maio, G. R., Perrucci, M. G., \& Ebisch, S. J. (2020). Sense or sensibility? The neuro-functional basis of the structural matching effect in persuasion. Cognitive, Affective, \& Behavioral Neuroscience, 20, 1-15. https://doi.org/10.31234/osf.io/pyr7s.

Bentler, P. M. (1990). Comparative fit indexes in structural models. Psychological Bulletin, 107(2), 238-246. https://doi.org/10.1037/ 0033-2909.107.2.238.

Brazil. Health Ministry. (2012). Resolução $n^{\circ} 466$, de 12 de dezembro de 2012. http://conselho.saude.gov.br/resolucoes/2012/Reso466.pdf

Brazil. Health Ministry. (2016). Resolução $n^{\circ}$ 510, de 07 de abril de 2016. http://conselho.saude.gov.br/resolucoes/2016/Reso510.pdf

Brazil. Health Ministry. (2020). Boletins epidemiológicos. https://www. saude.gov.br/boletins-epidemiologicos

Chen, F. F. (2007). Sensitivity of goodness of fit indexes to lack of measurement invariance. Structural Equation Modeling, 14(3), 464-504. https://doi.org/10.1080/10705510701301834.

Clarkson, J. J., Tormala, Z. L., \& Rucker, D. D. (2011). Cognitive and affective matching effects in persuasion: An amplification perspective. Personality and Social Psychology Bulletin, 37(11), 14151427. https://doi.org/10.1177/0146167211413394.

Falomir-Pichastor, J. M., Mugny, G., \& Berent, J. (2017). The side effect of egalitarian norms: Reactive group distinctiveness, biological essentialism, and sexual prejudice. Group Processess \& Intergroup Relations, 20(4), 540-558. https:// doi.org/10.1177/1368430215613843.

Francis, S. E., Noel, V. A., \& Ryan, S. L. (2019). A systematic review of the factor structure of anxiety sensitivity among children: Current status and recommendations for future directions. Child \& Youth Care Forum, 48(5), 603-632. https://doi.org/10.1007/s10566-01909502-y.

Garcia-Moreno, C., Heise, L., Jansen, H. A., Ellsberg, M., \& Watts, C. (2005). Violence against women. Science, 310(5752), 1282-1283. https://doi.org/10.1126/science.1121400.

Grant, J. S., \& Davis, L. L. (1997). Selection and use of content experts for instrument development. Research in Nursing \& Health, 20(3), 269-274. https://doi.org/10.1002/(sici)1098-240x(199706)20: 3<269::aid-nur9>3.0.co;2-g.

Hair, J., Black, W. C., Babin, B. J., Anderson, R. E., \& Tatham, R. L. (2006). Multivariate Data Analysis (6th edition). Upper Saddle River: Pearson Prentice Hall.

Hu, L.-T., \& Bentler, P. M. (1999). Cutoff criteria for fit indexes in covariance structure analysis: Conventional criteria versus new alternatives. Structural Equation Modeling, 6(1), 1-55. https://doi. org/10.1080/10705519909540118.
Kaiser, H. F. (1960). The application of electronic computers to factor analysis. Educational and Psychological Measurement, 20, 141151. https://doi.org/10.1177/001316446002000116.

Kang, L., Li, Y., Hu, S., Chen, M., Yang, C., Yang, B. X., Wang, Y., Hu, J., Lai, J., Ma, X., Chen, J., Guan, L., Wang, G., Ma, H., \& Liu, Z. (2020). The mental health of medical workers in Wuhan, China dealing with the 2019 novel coronavirus. Lancet Psychiatry, 7(3), e14. https://doi.org/10.1016/S2215-0366(20)30047-X.

Klackl, J., \& Jonas, E. (2019). Effects of mortality salience on physiological arousal. Frontiers in Psychology, 10, 1-14. https://doi.org/10. 3389/fpsyg.2019.01893.

Lovibond, P. F., \& Lovibond, S. H. (1995). The structure of negative emotional states: Comparison of the depression anxiety stress scales (DASS) with the Beck depression and anxiety inventories. Behaviour Research and Therapy, 33(3), 335-343. https://doi.org/ 10.1016/0005-7967(94)00075-U.

Mittal, M., \& Bhakar, S. S. (2018). Examining the impact of role overload on job stress, job satisfaction and job performance - A study among married working women in banking sector. International Journal of Management Studies, 2(7), 01-11. https://doi.org/10.18843/ijms/ v5i $2(7) / 01$

Mukhtar, S. (2020). Pakistanis' mental health during the COVID-19. Asian Journal of Psychiatry, 51(10), 21-27. https://doi.org/10. 1016/j.ajp.2020.102127.

Muthén, L. K., \& Muthén, B. O. (2017). Mplus User's Guide. Eighth Edition. Los Angeles: Muthén \& Muthén.

Nunnally, J. C., \& Bernstein, I. H. (1994). Psychometric theory. New York: McGraw-Hill.

Olatunji, B. O., \& Wolitzky-Taylor, K. B. (2009). Anxiety sensitivity and the anxiety disorders: A meta-analytic review and synthesis. Psychological Bulletin, 135(6), 974-999. https://doi.org/10.1037/ a0017428.

Oliver, N., Barber, X., Roomp, K., \& Roomp, K. (2020). Assessing the impact of the COVID-19 pandemic inSpain: Large-scale, online, self-reported population survey. Journal of Medical Internet Research, 22(9), 21-31. https://doi.org/10.2196/21319.

Pakpour, A. H., \& Griffiths, M. D. (2020). The fear of COVID-19 and its role in preventive behaviors. Journal of Concurrent Disorders, 2, 58-63 http://irep.ntu.ac.uk/id/eprint/39561/.

Parashar, P. (2020). Violence against women: Root causes and reasons for its continuity. Journal of Family and Adoption Law, 2(2), 9-17 http://lawjournals.stmjournals.in/index.php/jfal/article/view/351/ 280.

Putnik, D. L., \& Bornstein, M. H. (2016). Measurement invariance conventions and reporting: The state of the art and future directions for psychological research. Developmental Review, 41, 71-90. https:// doi.org/10.1016/j.dr.2016.06.004.

Sharma, S., Powers, A., Bradley, B., \& Ressler, K. J. (2016). Gene $\times$ environment determinants of stress- and anxiety-related disorders. Annual Review of Psychology, 67, 239-261. https://doi.org/10. 1146/annurev-psych-122414-033408.

Taylor, S., Landry, C. A., Paluszek, M. M., Fergus, T. A., McKay, D., \& Asmundson, G. J. G. (2020). Development and initial validation of the COVID stress scales. Journal of Anxiety Disorders, 72, 102232. https://doi.org/10.1016/j.janxdis.2020.102232.

Tucker, L., \& Lewis, C. (1973). A reliability coefficient for maximum likelihood factor analysis. Psychometrika, 38, 1-10. https://doi.org/ 10.1007/BF02291170.

Vignola, R. C. B., \& Tucci, A. M. (2014). Adaptation and validation of the depression, anxiety and stress scale (DASS) to Brazilian Portuguese. Journal of Affective Disorders, 155, 104-109. https:// doi.org/10.1016/j.jad.2013.10.031.

Wang, C., Pan, R., Wan, X., Tan, Y., Xu, L., Ho, C. S., \& Ho, R. C. (2020a). Immediate psychological responses and associated factors during the initial stage of the 2019 coronavirus disease (COVID-19) epidemic among the general population in China. International 
Journal of Environmental Research and Public Health, 17(5), 1729. https://doi.org/10.3390/ijerph17051729.

Wang, W., Xu, Y., Gao, R., Lu, R., Han, K., Wu, G., \& Tan, W. (2020b). Detection of SARS-CoV-2 in different types of clinical specimens. JAMA networks, 323(18), 1843-1844. https://doi.org/10.1001/jama. 2020.3786.

Wenham, C., Smith, J., \& Morgan, R. (2020). COVID-19: The gendered impacts of the outbreak. The Lancet, 395(10227), 846-848. https:// doi.org/10.1016/S0140-6736(20)30526-2.

WHO. World Health Organization. (2020). WHO Director-General's opening remarks at the media briefing on COVID-19. https:// www.who.int/dg/speeches/detail/who-director-general-s-openingremarks-at-the-media-briefing-on-covid-19\%2D\%2D-11-march2020
Wong, S. H., \& Chow, A. Y. M. (2017). A pilot study to validate measures of the theory of reasoned action for organ donation behavior. Death Studies, 42(4), 216-227. https://doi.org/10.1080/07481187. 2017.1334012

World Medical Association. (2001). World medical association declaration of Helsinki. Ethical principles for medical research involving human subjects. Bulletin World Health Organization, 79, 373-374.

Zandifar, A., \& Badrfam, R. (2020). Iranian mental health during the COVID-19 epidemic. Asian Journal of Psychiatry, 51, 101990. https://doi.org/10.1016/j.ajp.2020.101990.

Publisher's Note Springer Nature remains neutral with regard to jurisdictional claims in published maps and institutional affiliations. 